\title{
VALOR DO ESFREGAÇO NO DIAGNÓSTICO PER-OPERATÓRIO DOS TUMORES PROVENIENTES DE NEUROCIRURGIAS
}

\author{
LEILA CHIMELLI *, IVELISE DE SOUZA CAMPOS *
}

\begin{abstract}
RESUMO - A técnica do esfregaço no diagnóstico per-operatório de tumores provenientes de neurocirurgias vem sendo utilizada em diversos centros de neuropatologia. Com o objetivo de testar esta técnica, examinamos 137 esfregaços e correlacionamos os aspectos cito e histológicos. Analisamos 12 astrocitomas, 4 astrocitomas anapiásicos, 26 glioblastomas, 7 oligodendrogliomas, 7 ependimomas, 5 meduloblastomas, 8 schwannomas, 17 meningiomas, 13 adenomas de hipófise, 20 metástases e 18 tumores diversos e entidades não neoplásicas. Os resultados mostraram porcentual de acerto de $91,2 \%$, coincidente à literatura. $O$ conhecimento da locaízaça do tumor contribuiu para o acerto diagnóstico e o reconhecimento do tecido normal foi importante inclusive para orientar o neurocirurgião a localizar o tumor. Acreditamos que o esfregaço deva ser mais usado em nosso meio, não só pela simplicidade na sua realização mas também pela precisão diagnóstica.
\end{abstract}

PALAVRAS-CHAVE: diagnóstico citológico per-operatório, neurocirurgias, técnica do esfregaço.

Value of the gmear in the rapid diagnosis of tumors removed in neurosurgeries.

SUMMARY - The smear technique has been used for rapid diagnosis of tumors removed in neurosurgeries in many neuropathological centers. In order to assess the diagnostic accuracy of this technique, we examined 137 smearsi and made a cytohistological correlation. Our cases include 12 astrocytomas, 4 anaplastic astrocytomas, 26 glioblastomas, 7 oligodendrogliomas, 5 medulloblastomas, 8 schwannomas, 17 meningiomas, 13 pituitary adenomas, 20 metastatic tumors and 18 assorted tumors and non neoplastic lesions. The correct diagnosis was made in $91.2 \%$ of the cases which coincides to the literature. The knowledge of the localization and the characteristic morphologic features of some tumors were important for the diagnosis. The ability to recognize normal nervous tissue in smears was important even to help the surgeon to reach the tumor. We believe that the use of smears should be encouraged in our country in view of the simplicity of its preparation and accuracy of results.

KEY WORDS: intra-operative cytologic diagnosis, neurosurgeries, smear technique.

O neurocirurgião pode se beneficiar muito com o diagnóstico microscópico per-operatório, o que permite melhor planejamento da cirurgia, chegando muitas vezes a interrompê-la ou a adotar conduta totalmente diversa da prevista para um tumor quando processos não neoplásicos são diagnosticados. O diagnóstico rápido permite o planejamento da extensão do campo operatório, que, às vezes, não se estende além de um simples orifício de trépano ${ }^{1}$. Além disso, o diagnóstico per-operatório é muito utilizado na análise dos limites da neoplasia 5 .

Há duas técnicas de diagnóstico rápido: a congelação e o esfregaço. Nas biópsias por agulha, o material obtido é em geral escasso e amolecido, sendo quase impossível a obtenção de cortes satisfatórios em material congelado 11. Nestes casos o esfregaço é o ideal. E ainda, todo o material pode ser analisado, enquanto na congelação apenas um pequeno segmento é examinado, já que, em

* Departamento de Platologia, Universidade Federal Fluminense (UFF), Niterói. Aceite: 21-agosto-1992.

Dra. Leila Chimelli - Departamento de Patologia, Hospital Universitário Antônio Pedro Rua Marquês do Paraná 303 - 24030 Niterói RJ - Brasil. 
geral, só um ou dois cortes consecutivos são realizados. Além disso, o tempo gasto para fazer um esfregaço costuma ser menor que para um corte de conge. lação 1. Quanto à correlação com o diagnóstico definitivo, o esfregaço se equi. vale à congelação com porcentual de acerto de 87 a $93,41,3,12,43,45$, tendo sido um dos estudos realizado no Brasil 23. Com o objetivo de testar esta técnica, examinamos 137 esfregaços de tumores operados por neurocirurgiões e fizemos a correlação cito-histológica.

\section{MATBRIAL E MÉTODOS}

Analisamos citologicamente 137 casos e correlacionamos os diagnósticos ao exame histológico. Parte do material se originava do servico de neurocirurgia do Hospital Universitário Antônio Pedro da UFF e o restante de serviços de neurocirurgia externos. Em todos a técnica usada na realizaçăo dos esfregaços foi a mesma, sendo estes sempre analisados por nós no Departamento de Patologia. Nossa casuística inclui 12 astrocitomas, 4 astrocitomas anaplásicos, 26 glioblastomas, 7 oligodendrogliomas, 7 ependimomas, 5 meduloblastomas, 8 schwannomas, 17 meningiomas, 13 adenomas de hipófise, 20 tumores metastáticos e 18 tumores diversos o entidades não neoplásicas, assim agrupados porque o pequeno número de casos, não justificava formar grupos isolados. Este grupo inclui: 1 papiloma do plexo coróide, 1 germinoma, 1 neuroblastoma, 1 pineoblastoma, 1 tumor neuroectodermico primitivo, 1 fibromixossarcoma, 1 nevo de Ota, 2 malformações vasculares, 2 hemangioblastomas, 1 craniofaringioma, 1 plasmocitoma, 1 gliose reacional, 1 cordoma, 2 condrossarcomas e 1 cisto ósseo aneurismático.

Logo após a retirada do material, o fragmento era examinado macroscopicamente, os esfregaços realizados e o restante do material fixado em formol para estudo histológico. $O$ fragmento escolhido para o esfregaco media cerca de $1 \mathrm{~mm} 3$, era colocado próximo à extremidade de uma lâmina e, com outra lâmina colocada paralelamente sobre a que continha o fragmento, realizava-se o esfregaço. De 2 a 4 lâminas eram preparadas para cada caso $\theta$ imediatamente fixadas em álcool absoluto por 1 minuto. Após rápida lavagem em água corrente eram coradas com azul de toluidina a $1 \%$ por 1 minuto, lavadas em água corrente, passando rapidamente em álcool a 70\%, alcool absoluto (2x), xilol (2x), montando-se com bálsamo e laminula. Nos casos obtidos de serviços externos, os esfregaços eram realizados a fixados no momento da cirurgia, mas corados e examinados posteriormente apenas com o objetivo de testarmos nosso diagnóstico. O tempo médio gasto, desde o recebimento do material até a informação do diagnóstico ao cirurgiāo, era de aproximadamente 7 minutos, sendo um pouco maior em casos que requeriam mais tempo de análise da lâmina.

Admitindo-se que o diagnóstico histológico fosse o correto, correlacionamos os achados cito e histológicos. Eram considerados acertos os casos em que o exame histológico não acrescentou qualquer subsídio ao citológico e aqueles que, embora o diagnóstico citológico estivesse correto quanto a malignidade ou benignidade e quanto a linhagem histológica do tumor (ex. glioma), o exame histopatológico o enquadrou melhor em sua classificação. Foram considerados erros os casos em que foi o exame histológico que definiu o diagnóstico do tumor, seja porque o material era escasso ou nâo apropriado para a realização do esfregaço ou por dificuldade na sua interpretação e preferimos aguardar o exame histológico. Correlacionamos os acertos e erros à idade e ao sexo dos pacientes e com a localização dos tumores.

\section{RESULTADOS}

Houve coincidência de $91,24 \%$ dos diagnósticos citológicos e histológicos (125 casos). Os erros correram em 12 casos $(8,76 \%), 4$ deles por material escasso ou não apropriado para 0 esfregaço e preferimos aguardar o exame histológico. Os demais foram por dificuldade na interpretação do esfregaço. O material escasso em alguns de nossos casos deveu-se ao fato de serem oriundos de serviços externos, trazidos já fixados e não podíamos preparar maís esfregaços. Por vezes, observamos que o esfregaço era constituído de tecido nervoso normal - orientamos o cirurgião no sentido de que ainda não havia chegado no tumor. Os resultados estão sumiariados na Tabela 1 a a interpretação dos erros na Tabela 2. $O$ aspecto citológico dos tumores $e$ tema do trabalho a seguir.

Avaliamos a possivel influência do conhecimento do local dos tumores na correlação cito-histológica e observamos que o maior número de acertos ocorreu em tumores da região selar e têmporo-parietal $(\mathbf{1 0 0 \%})$, seguidas da fossia posterior (96,5\%), da região frontal (88,3\%) e de outras localizações $(87,5 \%)$. O conhecimento do sexo (83 homens e 54 mulheres) năo interferiu no diagnóstico e o conhecimento da idade (média de 42 anos), contribuiu para 0 diagnóstico em alguns casos, principalmente em crianças. 
Tabela 1. Correlação cito-histológica por grupos de tumores.

\begin{tabular}{|c|c|c|c|c|}
\hline & No casos & $\%$ acertos & $\begin{array}{l}\text { No erros } \\
\text { (casos) }\end{array}$ & Causa \\
\hline Tumores Astrociticos * & 42 & 92,9 & 3 & $\begin{array}{l}1 \mathrm{p} / \text { material escasso } \\
2 \mathrm{p} / \text { dificuldade diagnóstica }\end{array}$ \\
\hline Oligodendrogliomas & 7 & 85,7 & 1 & material escasso \\
\hline Ependimomas & 7 & 85,7 & 1 & dificuldade diagnóstica \\
\hline $\begin{array}{l}\text { Tumores neuroectodérmicos } \\
\text { primitivos }{ }^{* *}\end{array}$ & 8 & 100 & - & $\longrightarrow$ \\
\hline Schwannomas & 8 & 87,5 & 1 & material escasso \\
\hline Meningiomas & 17 & 100 & - & 一 \\
\hline Adenomas hipofisários & 13 & 100 & - & 一 \\
\hline Tumores metastáticos & 20 & 90 & 2 & $\begin{array}{l}1 \mathrm{p} / \text { material escasso } \\
1 \mathrm{p} / \text { dificuldade diagnóstica }\end{array}$ \\
\hline $\begin{array}{l}\text { Outros tumores e patologias } \\
\text { nāo neoplásicas }\end{array}$ & 18 & 71,4 & 4 & dificuldade diagnóstica \\
\hline
\end{tabular}

(*) englobam os astrocitomas, astrocitomas anaplásicos e glioblastomas multiformes; (**) englobam meduloblastoma, pineoblastoma e neuroblastoma.

Tabela 2. Correlação cito-histológica nos casos de erro.

\begin{tabular}{|c|c|c|}
\hline Diagn. Citológico & Histologia & Erro/causa do erro \\
\hline Astrocitoma anaplásico & Astrocitoma & $\begin{array}{l}\text { Tumor benigno interpretado como } \\
\text { maligno }\end{array}$ \\
\hline Aguardar histologia & Astrocitoma anaplásico & Material escasso na lâmina \\
\hline Astrocitoma & Glioblastoma & $\begin{array}{l}\text { Tumor maligno interpretado como } \\
\text { benigno }\end{array}$ \\
\hline Aguardar histologia & Oligodendroglioma & Material escasso na lâmina \\
\hline Aguardar histologia & Ependimoma & $\begin{array}{l}\text { Dificuldade na interpretacão } \mathrm{mi} \\
\text { croscópica }\end{array}$ \\
\hline Aguardar histologia & Schwannoma & Material escasso na lâmina \\
\hline Meningioma fibroblástico & Fibromixossarcoma & $\begin{array}{l}\text { *Tumor de baixo grau de maligni- } \\
\text { dade interpretado como be } \\
\text { nigno }\end{array}$ \\
\hline Aguardar histologia & Nevus de Ota & $\begin{array}{l}\text { Dificuldade na interpretação mi } \\
\text { croscópica }\end{array}$ \\
\hline Gangliocitoma ou astrocitoma & Angioma venoso & $\begin{array}{l}\text { Malformacão vascular interpreta } \\
\text { da como tumor benigno }\end{array}$ \\
\hline Condroma & Condrossarcoma & $\begin{array}{l}\text { Tumor maligno interpretado co- } \\
\text { mo benigno }\end{array}$ \\
\hline Aguardar histologia & Carcinoma metastático & Material escasso na lâmina \\
\hline Meningioma com anaplasia & $\begin{array}{l}\text { Metástase de carcinoma } \\
\text { em meningioma }\end{array}$ & $\begin{array}{l}\text { *Dificuldade na interpretação mi } \\
\text { croscópica }\end{array}$ \\
\hline
\end{tabular}

(*) Casos de difícil diagnóstico também na histologia. 


\section{COMENTARIOS}

O porcentual de diagnósticos cito e histológicos coincidentes em nossos casos foi 91,24, sendo próximo ao de outros autores: 94\% em 187 biópsias por agulha 13; 93\% em 216 biópsias em cirurgias amplas ou por agulha 3; 93,4\% numa série de 609 espécimes $1 ; 92 \%$ em 84 casos de biópsias por agulha a mão livre 12; 90,6\% em 198 tumores neuroepiteliais $23 ; 87 \%$ em 112 biópsias estereotáticas 25.

Quanto à localização, só tivemos 1 erro dentre os 28 tumores da fossa posterior. Segundo Marshall e Jennett 14, que acertaram todos os tumores desta região, é possível que o número limitado de tipos histológicos que incidem nesta região torne mais fácil o diagnóstico. O mesmo observamos nos tumores da região selar $(100 \%$ de acerto). O maior número de erros ocorreu no grupo de outras localizações que incluiu variedade maior de tumores.

Quanto aos tipos histológicos dos tumores observamos que nos astrocíticos o porcentual de acerto foi $\mathbf{9 2 , 8}$. Os erros ocorreram em função de tecido repre. sentativo do tumor estar ou não incluído nos esfregaços, o que pode ser devido em parte à escolha inadequada dos fragmentos $9,13,18$ ou a escassez de material recebido para o esfregaço, como referido por Torres e Collaço 23 , interferindo na graduação histológica, sem no entanto alterar a conduta cirúrgica. Willems e Alva-Willems 25 referiram cerca de $18 \%$ de erros nos astrocitomas malignos por se incluir nos esfregaços grande quantidade de tecido necrótico, o que ocorreu em um de nossos casos de glioblastoma. Noutro o material era insuficiente para o diagnóstico citológico.

Acertamos 6 dos 7 casos $(85,7 \%)$ de oligodendrogliomas. Em um, o esfregaço tinha pouco material não permitindo o diagnóstico. Alguns simulavam adenomas de hipófise, porém a localização do tumor, fora da região selar, orientou o diagnóstico 13,14 .

Em $6(85,7 \%)$ dos 7 ependimomas, o diagnóstico foi correto. Em um, pre. ferimos aguardar o exame histológico e a dificuldade na interpretação do esfre. gaço deveu-se à ausência do arranjo celular perivascular ou em rosetas, apesar dos prolongamentos fibrilares lembrarem a origem glial do tumor.

Agrupamos o meduloblastoma, o neuroblastoma e o pineoblastoma, por serem tumores neuroectodérmicos primitivos com aspecto citológico semelhante $1,8,18,24$. Acertamos $100 \%$ dos casos.

Apesar da consistência firme, é surpreendente a possibilidade de se diag. nosticar schwannomas em esfregaços 1,24,25: diagnosticamos 7 dos 8 casos $(87,5 \%)$. Em um, o diagnóstico não foi possível por escassez de material na lâmina. Noutro, houve dúvida entre schwannoma e astrocitoma pilocítico, por tratar-se de lesão cística na fossa posterior de paciente jovem. O esfregaço exibia prolongamentos fibrilares, mas optamos pelo diagnóstico de schwannoma. Tal dificuldade também tem sido relatada 14,15 .

Examinamos 17 meningiomas, todos com arranjo no esfregaço bastante característico, apesar dos vários tipos histológicos desses tumores e da consistência firme de alguns deles. Nossos resultados ( $100 \%$ de acerto) são coincidentes aos da literatura 3,24 .

O diagnóstico foi correto em todos os 13 adenomas de hipófise, o que atribuimos não só ao aspecto morfológico clássico 1,2,21,24, mas, também, à infor. mação da localização do tumor $3,20,25,27$. Observamos tecido hipofisário normal em poucos casos, o que é importante na diferenciação de adenomas hipofisários 1,26 .

Em $18(90 \%)$ dos 20 tumores metastáticos houve concordância cito-histológica. Um dos dois erros foi devido a ser o material escasso. O outro tratava-se de carcinoma metastático desenvolvendo-se em meningioma pré-existente, no esfregaço sendo observado o meningioma e algumas células anaplásicas. Há relatos de dificuldade em diferenciar alguns glioblastomas multiformes e astrocitomas anaplásicos de metástases pouco diferenciadas 1,4,7,13-15,21, podendo-se lançar mão de imuno-histoquímica 7.

No grupo que englobava os outros tumores e patologias não neoplásicas o porcentual de acerto foi de 71,4. Acreditamos que o maior número de erros 
deveu-se, em parte, à raridade de alguns tumores e ao fato de nem todos serem do sistema nervoso, sendo obtidos de laminectomias, com consistência não apropriada para o esfregaço, resultando em lâminas de má qualidade. Neste grupo, o papiloma do plexo coróide, o germinoma, o plasmocitoma, o cordoma, o craniofaringioma e o hemangioblastoma foram facilmente diagnosticados. Os dois últimos não propiciam bons esfregaços mas a informação da localização contribui para o diagnóstico 1,9. O fibromixossarcoma constituiu um problema diag. nóstico: a localização e o fato de ser bem delimitado e encapsulado sugeriam meningioma, mas o aspecto citológico não foi convincente. Após opiniões diver. sas, o tumor foi diagnosticado no exame histológico, como fibromixossarcoma de baixo grau de malignidade. A grande quantidade de pigmentos no nevo de Ota nos fez pensar em melanoma, mas faltavam mitoses e pleomorfismo 6 , e preferimos aguardar o exame histológico. Não tivemos dificuldades em um dos casos de malformação vascular o que não aconteceu no outro, já que sugerimos astrocitoma ou gangliocitoma no esfregaço, talvez pela presença de astrócitos reacionais e células próprias ao hipocampo (local do tumor). Erro similar foi referido por Marshall et al.13, ao diagnosticarem um astrocitoma pela presença de células hipercromáticas consideradas neoplásicas, identificadas no exame histológico como neurônios da fascia dentata do hipocampo, não havendo tumor. Em um dos casos de condrossarcoma fizemos o diagnóstico de cordoma que tem semelhanças com o condrossarcoma, mas detalhes citológicos e de localização podem ajudar na diferenciação ${ }^{1,26}$. O diagnóstico diferencial do cisto ósseo aneurismático foi com tumor de células gigantes e a confirmação foi feita no exame histológico. No caso em que o tecido era representado por gliose reacional optamos por informar ao cirurgião que, embora o tecido fosse anormal, não havia neoplasia.

As opiniões diferem quanto ao valor das técnicas de diagnóstico rápido. Há os que defendem o uso da congelação 16 por acharem que, com o esfregaço, se perde a arquitetura tecidual e que os fragmentos de consistência endurecida impossibilitam a realização de esfregaços de boa qualidade. Outros preferem o esfregaço 1,14,17,23 por ser: menor o tempo requerido no seu preparo; o resultado comparável ao da congelação, conseguindo-se resultados precisos com pequenos fragmentos; método portátil, de fácil preparo. Alguns sugerem o uso simultâneo das duas técnicas, como um meio diagnóstico mais seguro 19,26.

Acreditamos que a experiência com as técnicas de diagnóstico rápido é fundamental, devendo o patologista estar preparado para o diagnóstico pelo esfregaço, caso o material enviado para exame não permita a congelação. Em virtude de dispormos em nosso Departamento das duas possibilidades, preferimos realizar os esfregaços por sua rapidez e bom porcentual de acertos, usando a congelação nos poucos casos em que o material é inadequado para esfregaço. Lembramos que, mesmo em esfregaços, quando o material é escasso e que a parte representativa do tumor tenha sido usada na sua realização, é possível realizar técnicas imuno-histoquímicas para caracterizar as células e conciuir o diagnóstico $10,22,23$.

O diálogo do patologista com o cirurgião é fundamental e deve, sempre que possível, ocorrer antes da cirurgia pois, nos casos em que existam dificuldades na interpretação citológica, dados clínicos, radiológicos, macro e microscópicos serão ponderados na decisão diagnóstica.

\section{REFERENCIAS}

1. Adams JH, Graham DI, Doyle D. Brain biopsy: the smear techniques for neurosurgical biopsies. London: Chapman and Hall, 1981.

2. Balhuizen JC, Bots GThAM, Schaberg A. The value of cytology in the diagnosis of nypophyselal tumors. Acta Cytol 1974, 18:370-375.

3. Beckerley BB, Adams JH, Doyle D, Graham DI, Harper CG. The smear technique in the diagnosis of neurosurgical biopsies. N Z Med J 1978, 87:12-15.

4. Cahill EM, Hidvegi DF. Crush preparations of lesions of the central nervous system: a useful adjunct to the frozen section. Acta Cytol 1985, 29:279-285.

5. Daumas-Duport C, Scheithauer BW, Kelly PJ. A histologic and cytologic method for the spatial definition of gliomas. Miayo Clin Proc 1987, 62:435-449.

6. Enriquez R, Egbert B, Bullock J, Haven W. Primary malignant melanoma of central nervous system. Arch Pathol 1973, 95:392-395. 
7. Galloway PG, Roessmann U. Anaplastic astrocytoma mimicking metastatic carcinoma. Am J Surg Pathol 1986, 10:728-732.

8. Gandolfi A. The cytology of cerebral neuroblastoma. Acta Cytol 1980, 24:344-346.

9. Jane JA, Bertrand $G$. A cytological method for the diagnosis of tumors affecting the centrial nervous system. J Neuropathol Exp Neurol 1962, 21:400-409.

10. Laing RW, Smith CML. A useful technique for improving the diagnostic yield from single stereotactic biopsies of brain. Neuropathol Appi Neurobiol 1990, 16:529.

11. Larson CP, Reberger CC. Smear method for rapid diagnosis of neurosurgical material. Am J Clin Path 1952, 22:916.

12. Liwnicz BH, Henderson KS, Mazukawa T, Smith RD. Needle aspiration cytology of intracranial lesions: a review of 84 cases. Acta cytol 1982, 26:779-786.

13. Marshall LF, Adams H, Doyie D, Graham DI. The histological accuracy of the smear technique for neurosurgical biopsies. J Neurosurg 1973, 39:82-88.

14. Marshall LF, Jennett B. Smear biopsy in neurosurgical diagnosis. Arch Neurol 1973, $196033: 471-479$.

15. McMenemey WH. An appraisal of smear-diagnosis in neurosurgery. Am $J$ Clin Pathol $1960,33: 471-479$.

16. Miguel J, Calvo W, Rubinstein LJ. A simple and rapid stain for the biopsy diagnosis of brain tumors. J Neuropathol Exp Neurol 1968, 27:517-523.

17. Morris AA. The use of smear technique in the rapid histological diagnosis of tumors of the central nervous system. Description of a new staining method. J Neurosurg $1947,4: 497-504$.

18. Ostertag CB, Mennel HD, Kiessling IM. Stereotactic biopsy of brain tumors. Surg Neurol 1980, 14:275-283.

19. Owings RM. Rapid cytologic examination of surgical specimens: a valuable technique in the surgical pathology laboratory. Human Pathol 1984, 15:605-614.

20. Papo I, Beduschi A, D'Andrea F. Il metodo per schiacciamento e striscio nella diagnosi peroperatoria dei tumori endocranici. Acta Neurol (Napoli) 1956, 11:1182-1194.

21. Papo I, Colombo F. Possibilità e limite dell'esame estemporaneo per shiacciamento e striscio nella diagnosi peroperatoria dei tumori endocranici. Minerva Neurochirurg 1959, 3:134-147.

22. Taratuto AL, Sevlever G, Piccardo $P$. Clues and pitfalls in stereotactic biopsy of the central nervous system. Arch Path Lab Med 1991, 115:596-602.

23. Torres LFB, Collaço LM. Diagnóstico citopatológico de tumores neuroepiteliais pela técnica do esfregaço. Arq Neuro-Psiquiat (São Paulo) 1992, 50:173-179.

24. Watson CW, Hadju SI. Cytology of primary neoplasms of the central nervous system. Acta Cytol 1977, 21:40-47.

25. Willems JC, Alva-Willems JM. Accuracy of cytologic diagnosis of central nervous system neoplasms in stereotactic biopsies. Acta Cytol 1984, 28:243-249.

26. Yashon D, Jane JA. Central nervous system tissue cytology. Progr Exp Tumor Res 1972, $17: 346-362$.

27. Zaharapoulos P, Wong JY. Cytology of common primary midline brain tumors. Acta Cytol 1980, 24:384-390. 